\title{
Trivium
}

Revue franco-allemande de sciences humaines et sociales - Deutsch-französische Zeitschrift für Geistesund Sozialwissenschaften

15 | 2013

La science pense en plusieurs langues

\section{"Lost in translation » : réflexions sur la traduction et le dialogue interculturel}

\section{Bernd Stiegler}

Traducteur : Claudine Layre et Anne-Emmanuelle Fournier

\section{(2) OpenEdition}

Journals

Édition électronique

URL : http://journals.openedition.org/trivium/4704

DOI : $10.4000 /$ trivium.4704

ISSN : 1963-1820

Éditeur

Les éditions de la Maison des sciences de l'Homme

Référence électronique

Bernd Stiegler, « «Lost in translation » : réflexions sur la traduction et le dialogue interculturel », Trivium [En ligne], 15 | 2013, mis en ligne le 09 décembre 2013, consulté le 10 décembre 2020. URL http://journals.openedition.org/trivium/4704 ; DOI : https://doi.org/10.4000/trivium.4704

Ce document a été généré automatiquement le 10 décembre 2020.

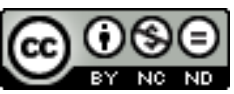

Les contenus des la revue Trivium sont mis à disposition selon les termes de la Licence Creative Commons Attribution - Pas d'Utilisation Commerciale - Pas de Modification 4.0 International. 


\title{
« Lost in translation » : réflexions sur la traduction et le dialogue interculturel
}

\author{
Bernd Stiegler \\ Traduction : Claudine Layre et Anne-Emmanuelle Fournier
}

\section{NOTE DE L'ÉDITEUR}

Nous remercions M. Bernd Stiegler ainsi que la maison d'édition Gunter Narr de nous avoir accordé l'autorisation de traduire ce texte pour le présent numéro. Wir danken Bernd Stiegler sowie dem Gunter Narr Verlag für die freundliche Genehmigung, diesen Artikel in französischer Übersetzung zu publizieren.

1 "Lost in Translation ", le dernier film de Sofia Coppola, fille du metteur en scène américain Francis Ford Coppola, remporte actuellement un grand succès auprès des spectateurs et des critiques. Ce succès s'explique en grande partie par la représentation convaincante d'une étrangeté, dont la dimension la plus évidente dans le film est celle qui naît de la confrontation à une autre culture, mais qui va de pair avec l'expérience d'une étrangeté dans les relations avec l'entourage immédiat et avec celle de l'étrangeté à soi-même. L'étrangeté de l'autre culture, ici celle du Japon, véhiculée sous une forme des plus hyperboliques, voire stéréotypées, à travers des images à l'exotisme désormais classique, est le reflet de l'expérience d'une étrangeté qui s'est nichée au cœur même du proche et du familier. Et ce que nous voyons d'étranger nous est déjà bizarrement familier, en raison d'une longue tradition de transmission culturelle de l'altérité à laquelle toute représentation de l'étranger peut recourir. Lorsqu'il s'agit de représenter l'étrangeté du Japon, c'est un fait acquis que les néons publicitaires et leurs idéogrammes jouent un rôle tout aussi éminent que les bars à karaoké et les temples zen. Toutes ces formes de mise en scène du regard étranger ont déjà été expérimentées et ont fait plus d'une fois leurs preuves. Elles font partie d'une iconologie cinématographique et littéraire qui peut recourir à toute une panoplie d'images. Dans 
le domaine des sciences littéraires et culturelles, cette relation a été largement analysée ${ }^{1}$ sous l'appellation d'«imagologie ». Les traductions s'insèrent d'ailleurs elles aussi dans ce programme de mise en scène de l'étrangeté qui s'ancre dans la culture de l'observateur et qui en dit finalement plus long sur cette dernière que sur la culture étrangère elle-même, ou qui révèle à la rigueur l'image figée et opaque que l'on se fait de cette autre culture, qui résiste en définitive à toute perception. Il en va de même pour les conclusions de la recherche en imagologie qui, non sans frustration mais à travers des observations indiscutables, constate de manière générale la pérennité des préjugés allemands sur la France et vice-versa, pour ensuite miser sur les nouvelles images que la pensée européenne pourrait faire naitre. Mais on ne ferait alors que continuer l'ancien jeu avec de nouvelles cartes: à la place de la France ou de l'Allemagne, on aurait les États-Unis² ${ }^{2}$ les pays africains ou précisément le Japon ...

2 Le film «Lost in translation » est un bon exemple de ce phénomène : il a été nominé aux Oscars uniquement parce que la représentation qu'il donne de l'étranger fait déjà partie d'un répertoire d'images connu des Américains. "Lost in translation" n'est pourtant pas un film sur le Japon, ni même un film sur l'image américaine du Japon, mais un film sur la relation entre un père de famille marié, entre deux âges, et une jeune femme mariée sans enfant, une relation dont les paramètres sont largement déterminés par la culture américaine (et peut-être aussi européenne). Nous évoluons sur le terrain familier d'une vision occidentale des choses. Aucune traduction de roman japonais n'y changerait rien, au contraire : la traduction s'inscrirait dans cette stratégie visant à mettre en scène l'étrangeté et à la transposer peu à peu dans sa propre culture. Même ce qui est étranger y a une place, un lieu prévu. Dans l'histoire de la littérature et de ses traductions, on pourrait sur ce point citer une série d'exemples, allant des classiques de la philosophie extrême-orientale aux "Mille et une nuits » dans le contexte de l'orientalisme, en passant par la traduction de récits mythiques venant de peuples et de pays étrangers ${ }^{3}$. Dans les sciences littéraires et culturelles, les colonial et postcolonial studies partent de tels phénomènes et analysent le regard culturellement et historiquement conditionné sur ce qui est étranger, regard qui ramène toujours l'étrangeté à une partie du monde connu ${ }^{4}$.

3 Dans « Lost in translation », ce qui est étranger nous est familier, car présenté sous une forme d'étrangeté que nous connaissons depuis longtemps et qui, si l'on y regarde de plus près, est si figée et en même temps si aléatoire qu'elle ne tolère aucune traduction. En effet, ce qu'il y aurait à traduire ne serait que banalité: des panneaux de signalisation, des néons publicitaires, des conventions sociales et des formes de loisirs courantes en Extrême-Orient. Et l'évidence de l'étrangeté est aussi forte que la connaissance de la signification des éléments qui échappent prétendument à notre culture. L'étrangeté est ici une mise en scène qui, en dépit de toute traduction qui pourrait être donnée, n'a rien d'autre en vue que la culture propre à l'observateur - en l'occurrence la nôtre. Ce premier modèle d'étrangeté, qui est aussi un modèle de traduction, nous pouvons le qualifier de projection de notre propre étrangeté. C'est une étrangeté familière.

4 Par opposition, dans un texte de 1970 qui fait presque figure de classique aujourd'hui, Roland Barthes rêve encore d'un monde sur lequel notre sensation de familiarité se fracasse, d'une langue intraduisible, d'un Empire des signes où une seule attitude est possible : «descendre dans l'intraduisible ${ }^{5}$ ». Roland Barthes justifie ce rêve par le souhait d'échapper aux contraintes de la culture occidentale, notamment aux contraintes 
conceptuelles qui nous ont été transmises depuis l'Antiquité grecque et qui continuent à déterminer nos modes de pensée, comme l'a souligné en particulier la théorie philosophique depuis Heidegger. Pour Barthes, cette autre culture qu'est le Japon doit provoquer en nous une sensation d'étrangeté face à notre propre culture, nous arracher à la "clôture " d'une "époque historico-métaphysique ${ }^{6}$ ", selon la formule de Derrida. Foucault lui aussi, et même Heidegger, ont chacun entrepris un voyage au Japon dont ils ont fait le récit dans des textes qui nous paraissent aujourd'hui bizarres, quelque peu erratiques, et dans lesquels l'insolite relation entre le proche, voire le très proche, et le lointain, voire le très lointain, joue un rôle décisif. Ainsi, c'est au Japon que Heidegger se sentait le mieux compris ${ }^{7}$; pour lui, le Japon était un lieu et une tradition où il pouvait vivre concrètement la mise entre parenthèses de sa propre tradition, comme il l'avait tenté en Europe 8 . Toutefois, cette reconnaissance de l'altérité court foncièrement le danger de retomber dans une pure projection de ce qui nous est propre, une projection «narcissique » de nos propres désirs, ne serait-ce que celui de sa propre théorie.

5 Cette deuxième façon de concevoir l'étrangeté peut être qualifiée d'utopie de l'intraduisible: elle est production d'une distance à notre propre culture, qui refuse cependant obstinément de perdre sa familiarité. C'est pour cela que la théorie philosophique élabore toute une série de processus, théories ou perspectives herméneutiques qui examinent précisément cette question et tentent de créer précisément cette distance. L'un de ces processus est par exemple la théorie (ainsi que la pratique philologique, philosophique et parfois aussi performative) de la destruction, qui englobe toujours aussi des questions de traduction. Toute la théorie et la pratique de l'avant-garde européenne, du futurisme et du dadaïsme aux théoriciens comme Ernst Jünger et Walter Benjamin ${ }^{9}$, en passant par le surréalisme et le Bauhaus, ont été fortement inspirées par une poétique de la destruction qui elle aussi présente de nombreux rapports avec la traduction. Et si Martin Heidegger conçoit de son coté la pratique de la lecture comme destruction résolue de la métaphysique occidentale et si son "retour " aux présocratiques a aussi essentiellement pour but de parvenir en amont des fondements de cette métaphysique, cette conception a également des conséquences pour la traduction et sa théorie. Cela ressort aussi bien à travers l'attention ostensible que Heidegger porte aux questions étymologiques que dans sa pratique spécifique de l'herméneutique (telle qu'elle apparait dans ses traductions de concepts grecs, comme sa célèbre interprétation du mot aléthéia, ainsi que dans ses textes tardifs où les interprétations étymologiques se voient constamment attribuer une signification philosophique prépondérante); ou encore dans ses propres traductions des textes et concepts classiques, qui cherchent à se distancer de la tradition occidentale tout en s'efforçant de sonder cet écart.

Il en va de même du côté français avec Jacques Derrida, dont l'œuvre présente un grand nombre de rapports avec la théorie de la traduction et qui ne nie pas sa proximité philosophique avec Martin Heidegger, ${ }^{10}$ mais aussi avec des écrivains comme Raymond Queneau, les surréalistes ou Henri Michaux (qui, comme Queneau, fut proche des surréalistes pendant quelques années), ou encore des théoriciens comme Michel Foucault et bon nombre de membres du groupe Tel Quel, pour ne citer que quelques exemples. On pourrait donner ici de nombreux autres noms, y compris des figures de la littérature et de la théorie d'outre-Rhin. 
7 Tandis que dans son roman Les enfants du limon ${ }^{11}$, Queneau oppose les « fous littéraires » à la tradition occidentale, et que Foucault considère plus tard la folie comme une voix étrangère au sein de sa propre culture (ce que montrent Histoire de la folie à l'âge classique $^{12}$ et nombre de ses essais ${ }^{13}$ ), s'enthousiasmant de son côté pour les «fous littéraires", on trouve chez Michaux une réflexion complexe sur l'expérience des drogues, cette dernière visant à mettre en parenthèses les prescriptions culturelles de l' écriture ${ }^{14}$ à visée artistique ${ }^{15}$. Les surréalistes ont mis au point de nombreux procédés afin de se soustraire temporairement au pouvoir de leur propre culture, le plus connu étant l'écriture automatique ${ }^{16}$. Le groupe Oulipo, dont faisaient partie Queneau, Perec, Calvino et Roubaud, a entrepris des expériences similaires, mais avec de strictes prescriptions formelles ${ }^{17}$. Pour tous ces auteurs, l'étrangeté représente une tentative de distanciation vis-à-vis de leur propre culture et n'est en aucun cas réductible à cette dernière. Cette étrangeté est le rêve d'une autre culture, avec une histoire, une langue et des traditions singulières, un monde d'images et un univers de pensée différents.

Tandis que dans le premier modèle de l'étrangeté, et corrélativement de la traduction, l'étrangeté représentée était avant tout une projection de celle de l'observateur - pour laquelle existait quand même une place au sein de sa propre culture - le deuxième modèle est sans conteste plus radical, mais en même temps tout aussi aporétique. Ici, l'étrangeté est un contre-modèle de la culture d'appartenance et de cette culture en tant que telle. Par conséquent, la traduction est une entreprise délicate, puisque par essence contre-productive, sauf à la rigueur au sein d'une conception théorique où on lui attribue une place, dès lors que la théorie vise quelque chose de comparable. Ce second modèle radicalise l'expérience de l'étrangeté et la transforme en échappatoire de la tradition d'appartenance, ressentie comme oppressante avec ses codes historiques et culturels. Il ne s'agit pas ici d'un reflet de ce qui est familier, mais de sa contreimage, d'un contre-modèle de la culture de référence de l'individu, avec toutes les difficultés que cela entraîne. Il s'agit en fin de compte de la traduction et/ou de l'étrangeté comme utopie.

9 C'est entre ces deux positions, celle de la projection et celle de l'utopie, que se situe à mon avis le modèle de la traduction. Je défends ici la thèse selon laquelle la traduction sert moins à la compréhension culturelle qu'à la production d'une forme spécifique de l'étrangeté qui, lorsque la traduction est réussie, provoque des effets d'étrangeté dans les deux cultures, effets qui entraînent à leur tour une compréhension culturelle double et en même temps réciproque. Elle n'est ni étrangeté familière de l'étranger culturel mis en scène ni utopie d'une étrangeté qui servirait de négatif à l'horizon culturel connu.

10 Éclairons brièvement ce point en prenant pour exemple les traductions entre le français et l'allemand dans le domaine philosophique au $\mathrm{XX}^{\mathrm{e}}$ siècle. Comme l'a montré Vincent Descombes ${ }^{18}$, lorsqu'au début du $\mathrm{XX}^{\mathrm{e}}$ siècle, à côté de Nietzsche, les trois grands « H », Hegel, Husserl, Heidegger, influencent de façon prépondérante les principaux penseurs français - de Sartre à Derrida en passant par Foucault et Levinas - cela amène à des positions philosophiques qui non seulement ont peu de rapport, voire probablement aucun, avec la philosophie allemande mais qui en outre se différencient nettement des interprétations allemandes des œuvres de Hegel, Husserl et Heidegger. On pourrait certes objecter que certains théoriciens allemands de la littérature des années soixante-dix (je pense en particulier à Hans Robert Jauss et à Wolfgang Iser) et quelques rares philosophes comme Hans Blumenberg ont produit des travaux qui 
peuvent être considérés comme le "pendant allemand " des théories post- ou néostructuralistes françaises. Mais cela ne ferait que confirmer - avec seulement un léger "déphasage " - la constatation selon laquelle la traduction produit bel et bien des effets d'étrangeté, puisque les champs scientifiques sont tout à fait différents (le paradigme migre de la philosophie vers la théorie littéraire) et que la position de Blumenberg, en raison notamment de sa dimension "métaphorologique ${ }^{19}$ ", est pour sa part restée sans grand écho dans la philosophie de son époque. Il n'est donc pas étonnant que ce soit précisément les philosophes et théoriciens qui ont œuvré en faveur de l'échange culturel et théorique qui rencontrent des problèmes considérables à être reconnus comme des théoriciens importants dans le pays de l'autre. En raison de leur positionnement inédit dans un contexte théorique et discursif, leur contribution spécifique au débat est tellement éloignée de la position d'origine que la « retraduction » de la théorie pose d'immenses difficultés et que les théories de départ deviennent quasiment méconnaissables dans les pays qui leur ont donné le jour. La « traduction » d'une théorie vers un autre contexte culturel ou théorique exige en effet certaines accentuations qui sont très éloignées des interprétations supposées évidentes de la théorie d'origine et qui font obstacle à sa réception dans son propre pays. Donnons seulement trois exemples : si la réception du post- ou néo-structuralisme en Allemagne aurait eu sans Manfred Frank un visage tout autre ${ }^{20}$, la traduction de ses travaux n'a en revanche rencontré quasiment aucun écho en France (et aucun Français ne situerait Derrida dans la même tradition que Foucault, Barthes ou Lévi-Strauss et a fortiori ne les réunirait sous une "appellation" théorique commune). Dans le sens inverse, Rainer Rochlitz a contribué comme nul autre à la réception de Jürgen Habermas et de l'École de Francfort en France, mais n'a eu que peu de succès avec ses propres écrits en Allemagne. Quant à Jacques Bouveresse, en dépit du fait qu'il a considérablement contribué à la découverte de Wittgenstein en France avant d'acquérir une voix tout à fait autonome au sein de la philosophie analytique, il continue à être parfaitement inconnu en Allemagne. On pourrait continuer cette liste et l'élargir aussi à des exemples historiques. Ce qui est commun à tous ces cas, c'est que les tentatives de traduction (à entendre ici au sens large) doivent nécessairement procéder à de nouvelles contextualisations, par le biais desquelles les textes ou théories d'origine sont à la fois re- et décontextualisés de manière spécifique. Ce phénomène entraîne deux sortes d'effets d'étrangeté : d'une part apparaît soudain dans une tradition théorique une nouvelle voix, qui serait certainement restée muette et inaudible sans tentative de traduction et sans réception; d'autre part, en raison de cette nouvelle formulation, la théorie en devient parfois méconnaissable pour le pays d'origine. C'est une situation tout à fait paradoxale mais qui ne signifie en aucune façon la fin des efforts et des succès traductologiques, comme le montrent quelques exemples qui recourent à un horizon temporel plus large.

11 Prenons par exemple les cours d'Alexandre Kojève ${ }^{21}$ sur Hegel : ils ont joué un rôle prépondérant pour la réception de Hegel en France mais sont irréconciliables avec l'hégélianisme allemand de l'époque. De même, la traduction et l'interprétation que fait Jacques Derrida du texte de Husserl Ursprung der Geometrie (Introduction à l'origine de la géométrie) ont très peu à voir avec une phénoménologie d'origine allemande. Du point de vue inverse, la situation est quasiment semblable : Hegel, Husserl et Heidegger ne correspondent à aucune position connue et introduisent dans le milieu philosophique français une voix étrangère qui tire ses effets de cette étrangeté. (L'importance que peut prendre cette nouvelle voix est certes due à l'existence de traductions mais, fait 
curieux, elle ne le doit pas nécessairement à leur qualité. L'œuvre de Walter Benjamin a ainsi bénéficié d'une réception très dense en France malgré une traduction par Maurice de Gandillac qui était vraiment très éloignée du texte allemand ${ }^{22}$.

Deux décennies plus tard, on assiste en Allemagne à un mouvement en sens inverse : la philosophie contemporaine française fait actuellement l'objet d'une large réception. Ici aussi, aucune approche théorique connue, ni celle de la première ou de la deuxième École de Francfort, ni l'existentialisme allemand, ni le groupe "Poétique et herméneutique ", ni la philosophie analytique d'inspiration wittgensteinienne - pour ne citer que quelques exemples - ne correspondent aux positions de Derrida, Levinas, Barthes ou Foucault. Et là encore, des positions qui sont nettement séparées en France (quiconque a assisté à un séminaire sur Foucault en France en supposant à tort qu'il y serait aussi question de Derrida, Barthes ou de Lacan, comme ce serait naturellement le cas en Allemagne, sait de quoi je parle) prennent une nouvelle tournure et sont soudain censées être parentes. Du néo-structuralisme à la postmodernité en passant par le poststructuralisme, on invente sans cesse de nouvelles étiquettes supposées unifier les théories et dont les théoriciens ont le plus grand mal à se défaire. De même, environ une décennie plus tard, s'amorce un débat qui relie l'École de Francfort et la théorie foucaldienne du pouvoir ou qui lit Derrida avec Benjamin et Benjamin avec Derrida. Cela donne naissance à un dialogue singulier qui inclut dans une égale mesure proximité et éloignement, et ce des deux côtés de la traduction.

Nietzsche aus Frankreich (le Nietzsche français) : tel est le titre d'un ouvrage qui illustre ce phénomène de manière particulièrement exemplaire. Précisément, il nous présente un Nietzsche différent, mais avec une voix qui colore à son tour la réception allemande puis italienne (celle qui suscita tout de même l'édition des Euvres complètes par Colli et Montinari) d'un timbre qui lui manquait jusqu'ici : une raucité qui modifie le ton que l'on connaît sans lui ôter tout à fait sa familiarité.

\section{BIBLIOGRAPHIE}

Barthes, R. (1994) : «L’Empire des signes », in : id. : Euvres complètes, t. 2 : 1966-1973, éd. par

E. Marty, Paris, p. 742-831.

Blumenberg, H. (2006 [1997]) : Paradigmes pour une métaphorologie, trad. par D. Gammelin, Paris.

Blumenberg, H. (2010 [2002]) : L'imitation de la nature et autres essais esthétiques, trad. par

M. de Launay et I. Kalinowski, Paris.

Buchner, H. (éd.) (1989) : Japan und Heidegger, Sigmaringen.

Childs, P. / Williams, P. (1997) : An Introduction to Post-Colonial Theory, Londres.

Derrida, J. (1967) : De la grammatologie, Paris.

Derrida J. (1986) : De l'esprit, Paris;

trad. allemande par A. García Düttmann : Vom Geist. Heidegger und die Frage, Francfort-sur-le-Main, 1988. 
Descombes, V. (1979) : Le Même et l'autre : quarante-cinq ans de philosophie française (1933-1978),

Paris ;

trad. allemande par U. Raulff : Das Selbe und das Andere. Fünfundvierzig Jahre Philosophie in

Frankreich. 1933-1978, Francfort-sur-le-Main, 1981.

Foucault, M. (1961) : L'histoire de la folie à l'âge classique, Paris ;

trad. allemande par U. Köppen : Wahnsinn und Gesellschaft. Eine Geschichte des Wahns im Zeitalter der Vernunft, Francfort-sur-le-Main, 1969.

Foucault, F. (1994a) : « Michel Foucault et le zen : un séjour dans un temple zen », in : id. : Dits et écrits, t. 3 : 1976-1979, Paris, texte ${ }^{\circ} 236$.

Foucault, F. (1994b) : « Sept propos sur le septième ange », in : id. : Dits et écrits, t. 2 : 1976-1979, Paris, texte $n^{\circ} 73$.

Frank, M. (1983) : Was ist Neostrukturalismus?, Francfort-sur-le-Main.

Kojève, A. (1979) : Introduction à la lecture de Hegel, reprod. en fac-sim. de la 2e éd. de Paris, Gallimard, 1968 ;

trad. allemande par I. Fetcher et G. Lehmbruch : Hegel. Eine Vergegenwärtigung seines Denkens. Kommentar zur „Phänomenologie des Geistes“, 5e éd. augmentée, Francfort-sur-le-Main.

Landmann, A. (2003) : « Zeichenleere ». Roland Barthes interkultureller Dialog mit Japan, Munich.

Mc Kenzie, J. H. (1995) : Orientalism. History, Theory, and the Arts, Manchester.

Moore-Gilbert, B. (1997) : Postcolonial Theory. Contexts, Practices, Politics, Londres.

Offe, C. (2004) : Selbstbetrachtung aus der Ferne. Amerikareisen von Tocqueville, Weber und Adorno, Francfort-sur-le-Main.

Queneau, R. (1938) : Les enfants du limon, Paris.

Saïd, E. (1978) : Orientalism, Londres ;

trad. fr. par C. Malamoud : L'orientalisme. L'Orient créé par l'Occident, Paris, 1980.

Stiegler, B. (1998) : « La destruction et l'origine : Ernst Jünger et Walter Benjamin », Littérature, 112, décembre, p. 112-126.

\section{NOTES}

1. Voir les publications de H. Dyserinck, W. Leiner ou M. S. Fischer dans ce domaine.

2. Une série de publications existe également à ce sujet. Citons par exemple Offe (2004).

3. Voir l'ouvrage exemplaire et bien documenté d'E. Saïd (1978), devenu depuis une œuvre de référence. Voir aussi Mc Kenzie (1995).

4. Voir le panorama de ces phénomènes présenté dans Moore-Gilbert (1997) ; Childs / Williams (1997).

5. En français dans le texte [N.d.T.]. Barthes (1994), p. 748. Sur le texte de Barthes, voir Landmann (2003).

6. Voir Derrida (1967), p. 14.

7. Voir par exemple Buchner (1989).

8. Voir Foucault (1994).

9. Voir Stiegler (1998).

10. Sur ces deux points, voir Derrida (1986).

11. Queneau (1938). 
12. Foucault (1961).

13. Voir Foucault (1994b).

14. En français dans le texte [N.d.T.].

15. C'est un topos de la littérature sur les drogues et/ou produite sous leur influence, tout au moins au XXe siècle.

16. En français dans le texte [N.d.T.].

17. Voir les recueils de textes Oulipo. La Bibliothèque Oulipienne, Paris, 1978 sq. Sont rassemblées dans ces fascicules un grand nombre de publications individuelles plutôt courtes, en général éditées d'abord séparément.

18. Descombes (1979).

19. Voir Blumenberg (2006 [1997]) ; (2010 [2002]).

20. Voir Frank (1983), un ouvrage qui contribua à l'époque considérablement à faire découvrir la philosophie française contemporaine. Cependant, l'œuvre de M. Frank ne se limite pas à cette réflexion voire à cette importation théorique; il est sans conteste l'un des plus grands spécialistes mondiaux du préromantisme et de la théorie de la conscience de soi. A ce titre, la France aurait pu le « découvrir » aussi comme théoricien.

21. Kojève (1979).

22. Un échantillon de sa traduction des essais a révélé qu'il manque des parties de texte et que d'autres ne sont que des improvisations sur un thème benjaminien. Depuis, toutefois, Gallimard a publié une nouvelle traduction très précise dans une édition très soignée, prenant la relève de celle de Gandillac qui a été la référence pendant des décennies. Nous pourrions citer d'autres exemples, des deux côtés du Rhin.

INDEX

Mots-clés : Traduction, étrangeté, réception, contextualisation

Schlüsselwörter : Übersetzung, Fremdheit, Rezeption, Kontextualisierung

\section{AUTEURS}

\section{BERND STIEGLER}

Bernd Stiegler est professeur de littérature moderne allemande. Pour en savoir davantage, consulter la notice 